\title{
Entropy Inflection and Invisible Low-Energy States: Defensive Alliance Example
}

\author{
Yi-Zhi Xu ${ }^{1,4}$, Chi Ho Yeung ${ }^{2}$, Hai-Jun Zhou ${ }^{1,4,5}$, and David Saad ${ }^{3}$ \\ ${ }^{1}$ CAS Key Laboratory for Theoretical Physics, Institute of Theoretical Physics, \\ Chinese Academy of Sciences, Beijing 100190, China \\ ${ }^{2}$ Department of Science and Environmental Studies, \\ The Education University of Hong Kong, Hong Kong \\ ${ }^{3}$ Nonlinearity and Complexity Research Group, Aston University, Birmingham B4 7ET, United Kingdom \\ ${ }^{4}$ School of Physical Sciences, University of Chinese Academy of Sciences, Beijing 100049, China \\ ${ }^{5}$ Synergetic Innovation Center for Quantum Effects and Applications, \\ Hunan Normal University, Changsha 410081, China
}

(Dated: November 22, 2018)

\begin{abstract}
Lower temperature leads to a higher probability of visiting low-energy states. This intuitive belief underlies most physics-inspired strategies for addressing hard optimization problems. For instance, the popular simulated annealing (SA) dynamics is expected to approach a ground state if the temperature is lowered appropriately. Here we demonstrate that this belief is not always justified. Specifically, we employ the cavity method to analyze the minimum strong defensive alliance problem and discover a bifurcation in the solution space, induced by an inflection point in the entropy-energy profile. While easily accessible configurations are associated with the lower-free-energy branch, the low-energy configurations are associated with the higher-free-energy branch within the same temperature range. There is a discontinuous phase transition between the high-energy configurations and the ground states, which generally cannot be followed by SA. We introduce an energy-clamping strategy to obtain superior solutions by following the higher-free-energy branch, overcoming the limitations of SA.
\end{abstract}

Statistical physics associates the probability of visiting low-energy states with low temperatures. This has inspired the introduction of Metroplis-like algorithms [1, such as simulated annealing (SA), which sample lowenergy configurations while gradually decreasing the temperature $T$, to progress towards equilibrium configurations close to the ground states [2]. An implicit fundamental assumption in SA is that the configuration entropy $S(E)$ is a concave function of the energy $E$ so that higher inverse temperature $\beta$ ( $\equiv 1 / T)$ corresponds to lower $E$. In this work we show that for an important class of discrete-state systems, the entropy function is not always concave but is characterized by an inflection point that separates the concave higher-energy branch from the convex lower-energy branch (Fig. 1). Because low-energy configurations are associated with high microcanonical temperatures, they cannot be accessed by lowering the ambient temperature in a quasi-equilibrium manner. The convex section of $S(E)$ also violates results obtained for canonical ensembles, suggesting a discrepancy between canonical and microcanonical ensemble analyses, as in $3-$ 5]. Advanced multicanonical methods, 6 6 -10 that allow for an exchange between different temperatures will fail as well, being rooted in the Boltzmann-Gibbs equilibrium framework, while the entropy inflection means there must be a discontinuous phase transition between the ground state and high-energy configurations.

The exemplar optimization task adopted here is the minimum Strong Defensive Alliance (SDA) problem [11, a special case of finding substructures in a large graph [12 14. More specifically, one aims to identify the smallest group $A$ of vertices (the alliance) in the graph such that at least one half of the nearest neighbors of each alliance-vertex also belong to the alliance (Fig. 1). It is a nondeterministic polynomial hard (NP-hard) problem and has raised considerable interest among mathematicians [15 17]. In statistical physics the SDA is closely related to the concepts of self-sustained clusters [18 20 and metastable states [21, 22], which are important for understanding the slow dynamics in spin systems. The synergetic excitation of a SDA may also drive rare but catastrophic cascading processes in real-world complex networks 23]. In this paper we apply the cavity method of spin glasses 24 26] to the SDA problem. We find that the entropy function $S(E)$ is non-concave for relatively sparse graphs but recovers concavity when the graph becomes sufficiently dense. In addition, we develop a principled energy-clamping algorithm to construct nearly optimal alliance solutions. The insights gained in this study are applicable to a range of similar problems concerning
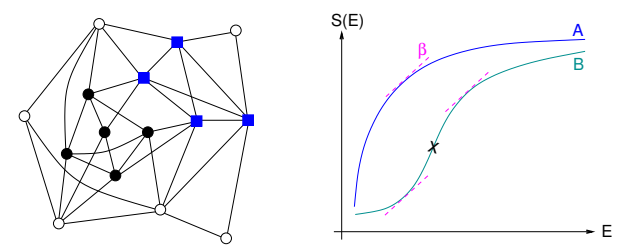

FIG. 1: (left) Two strong defensive alliance solutions for a small graph: the one denoted by filled circles has energy $E=5$; the other denoted by filled squares is the minimum alliance, $E=4$. (right) Two qualitatively different entropy curves $S(E)$ : curve A is concave, its slope $\beta(E)$ decreases with energy $E$; curve B is non-concave, it has an inflection point (' $\mathrm{X}$ ') at which the slope $\beta$ attains the maximum value. 
densely connected subgraphs.

Strong Defensive Alliance.- Given a graph $G$ of $N$ vertices and $M$ edges, a non-empty subset $A$ of vertices is regarded as an alliance if and only if at least half of the nearest neighbors of every vertex $i \in A$ are also in $A$. The minimum SDA problem aims to construct such an alliance of smallest cardinality, which requires a careful choice of vertices because SDA is a collective property of all vertices involved. For regular graphs in which every vertex has the same number $K$ of attached edges, the minimum alliance number is 2 if $K=1,2$ and it is equal to the graph's girth (the length of shortest loops) if $K=3,4$. But for all $K \geq 5$ the minimum SDA problem is intrinsically hard to solve, and the minimum alliance number is unknown and is difficult to bound 27. Here we apply methods and algorithms of statistical physics to tackle this challenging problem. For clarity we focus on regular random $(\mathrm{RR})$ graphs, in which every vertex is linked to $K$ randomly drawn vertices. The formulation is generic and can be applied to other degree profiles.

We cast the problem into a Hamiltonian form $E(\boldsymbol{c})=$ $\sum_{i=1}^{N} \delta_{c_{i}}^{1}$, where $c_{i}=1$ (the occupied state) if vertex $i$ belongs to the alliance and $c_{i}=0$ otherwise, and $\boldsymbol{c} \equiv\left(c_{1}, c_{2}, \ldots, c_{N}\right)$ denotes an occupation configuration of the $N$ vertices; the Kronecker symbol $\delta_{c}^{c^{\prime}}=1$ if $c=c^{\prime}$ and 0 otherwise. Let us denote by $\partial i$ the set of nearest neighbors of vertex $i$ and by $d_{i} \equiv|\partial i|$ its degree $\left(d_{i}=K\right.$ if $G$ is regular). Each vertex $i$ gives rise to a constraint on $c$ : if $c_{i}=1$ then $\sum_{j \in \partial i} c_{j} \geq d_{i} / 2$ must hold. Under these vertex constraints the partition function is

$$
Z(\beta)=\sum_{\boldsymbol{c} \neq \mathbf{0}} \prod_{i=1}^{N}\left[\delta_{c_{i}}^{0}+e^{-\beta} \delta_{c_{i}}^{1} \Theta\left(\sum_{j \in \partial i} c_{j}-\frac{d_{i}}{2}\right)\right],
$$

where the Heaviside function $\Theta(x)=1$ if $x \geq 0$ and 0 otherwise. The all-zero crystalline state $\mathbf{0} \equiv(0,0, \ldots, 0)$ has been excluded from the summation since it does not correspond to an alliance. Each satisfying configuration (alliance) $\boldsymbol{c}$ contributes a term $e^{-n \beta}$ to $Z(\beta)$, where $n \equiv$ $\sum_{i=1}^{N} c_{i}$ is the size of the alliance.

Simulated annealing.- We implement a Markov-chain Monte Carlo dynamics to explore the SDA configuration space, which includes both single-vertex flipping and the simultaneous flipping of a connected chain or tree of vertices (details in 28 ). The Monte Carlo simulation runs for $w_{0}$ time steps at each ambient inverse temperature $\beta$ (one step contains $N$ flipping trials selected by importance sampling which guarantees detailed balance [1, 29]), and then $\beta$ is increased by a constant value $\varepsilon$ (e.g., $\varepsilon=0.001)$. We run SA to identify SDA on two large RR graphs with degrees $K=3$ and $K=5$, and the results are shown in Fig. 2(a) and 2(b) respectively. In both cases, the average SDA relative size $\rho$ (i.e. the energy density) first decreases gradually with increasing $\beta$ as anticipated; but it then violently fluctuates between two distinct levels as illustrated in the inset of Fig. 2(a)

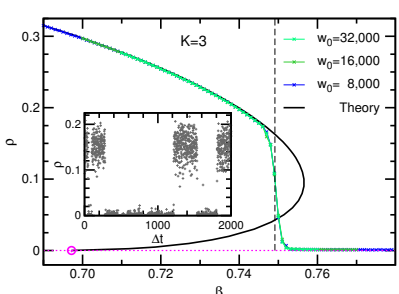

(a)

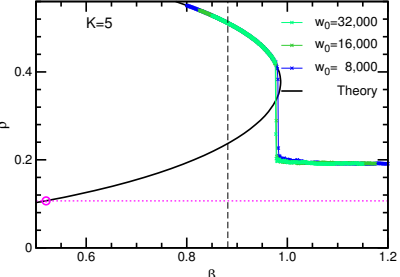

(b)
FIG. 2: The energy density $\rho$ of SDA identified by simulated annealing on a single RR graph of size $N=10^{4}$ and degree $K=3$ (a) or $K=5$ (b), as a function of ambient inversetemperature $\beta$. Evolution trajectories obtained at three different waiting times $w_{0}$ are shown. The solid lines represent the theoretical curves of $\rho(\beta)$; the dotted horizontal lines and the circles mark the theoretical value of minimum energy density and the corresponding theoretical $\beta$. SA can reach the predicted minimum SDA size for $K=3$ but not for $K=5$. Dashed vertical lines mark $\beta$ values at the predicted discontinuous phase transition. The inset shows the fluctuation of $\rho$ at $\beta=0.75$ for $K=3$, with $\Delta t$ being the elapsed simulation time starting from an initial equilibrium configuration.

when $\beta$ reaches a certain value $\beta_{S A}(\approx 0.75$ for $K=3$ and $\approx 0.98$ for $K=5$ ); finally it settles at a low level as $\beta$ further increases. These simulation trajectories indicate the existence of a discontinuous phase transition, which is surprising since we do not expect the low-energy and minimum SDA solutions to be qualitatively different from the higher-energy SDA solutions.

For RR graphs with $K=3$ and 4 , the minimum SDA are triangular loops, which are frequently visited by the SA dynamics after $\rho$ drops to $\rho \sim 1 / N$. Since SA also saturates at a low energy level for the instance of $K=5$ (Fig. 2p), one would naively claim the observed final value $\rho \approx 0.187$ to be the minimum energy density. However, it turns out that the true minimum energy density is much lower $(\approx 0.1067)$. Similar SA failures to visit low-energy configurations are observed on other graph instances [28. This might look unsurprising initially, since $\mathrm{SA}$ is well known to get trapped in metastable states if the low-energy configuration space fragments to an exponential number of disconnected ergodic domains [30, 31. However, our analysis does not support the emergence of such an explosive ergodicity-breaking phase transition at a high level of energy density [32 34] (additional discussions in [28]).

The performance of SA does not improve even in the presence of multiple-vertex flipping processes to guarantee ergodicity 28. We also implemented an ergodic SA algorithm with a relaxed Hamiltonian, whereby energy penalty terms rather than hard constraints are employed to penalize vertices which violate the SDA condition. The obtained results are similar to those observed in Fig. 2 $\mathrm{b}$, confirming the robustness of our simulation results 28 . It turns out that the peculiar sudden drop followed by 
jamming as experienced by SA is due to another important but rarely discussed reason: the entropy curve as function of $\rho$ has an inflection point.

Mean field theory.- Random sparse graphs are characterized by long loops that diverge with graph size $N$. This allows us to consider the neighborhood of single vertices $i$ as tree-like, and for the neighboring vertices $j \in \partial i$ as mutually independent in the absence of $i$. Under this BethePeierls factorization approximation [24-26], the marginal probability $q_{i}$ of vertex $i$ belonging to the alliance is

$$
q_{i}=\frac{e^{-\beta} \sum_{\boldsymbol{c}_{\partial i}} \Theta\left(\sum_{j \in \partial i} c_{j}-\frac{d_{i}}{2}\right) \prod_{j \in \partial i} q_{j \rightarrow i}^{c_{j}, 1}}{e^{-\beta} \sum_{\boldsymbol{c}_{\partial i}} \Theta\left(\sum_{j \in \partial i} c_{j}-\frac{d_{i}}{2}\right) \prod_{j \in \partial i} q_{j \rightarrow i}^{c_{j}, 1}+\prod_{j \in \partial i}\left(q_{j \rightarrow i}^{0,0}+q_{j \rightarrow i}^{1,0}\right)} .
$$

Here $\boldsymbol{c}_{\partial i} \equiv\left\{c_{j}: j \in \partial i\right\}$ denotes an occupation pattern of vertices in $\partial i$; and $q_{j \rightarrow i}^{c_{j}, c_{i}}$ is the probability of two nearest neighbors $i$ and $j$ being in states $c_{i}$ and $c_{j}$ simultaneously after lifting the constraint of vertex $i$. Following the same factorization approximation we obtain a closed set of selfconsistent equations for the cavity probabilities $q_{j \rightarrow i}^{c_{j}, c_{i}}$ :

$$
\begin{aligned}
q_{j \rightarrow i}^{0,0} & \equiv q_{j \rightarrow i}^{0,1}=\frac{1}{z_{j \rightarrow i}} \prod_{k \in \partial j \backslash i}\left(q_{k \rightarrow j}^{0,0}+q_{k \rightarrow j}^{1,0}\right), \\
q_{j \rightarrow i}^{1,0} & =\frac{e^{-\beta}}{z_{j \rightarrow i}} \sum_{c_{\partial j \backslash i}} \Theta\left(\sum_{k \in \partial j \backslash i} c_{k}-\frac{d_{j}}{2}\right) \prod_{k \in \partial j \backslash i} q_{k \rightarrow j}^{c_{k}, 1}, \quad(3) \\
q_{j \rightarrow i}^{1,1} & =\frac{e^{-\beta}}{z_{j \rightarrow i}} \sum_{\boldsymbol{c}_{\partial j \backslash i}} \Theta\left(\sum_{k \in \partial j \backslash i} c_{k}+1-\frac{d_{j}}{2}\right) \prod_{k \in \partial j \backslash i} q_{k \rightarrow j}^{c_{k}, 1},
\end{aligned}
$$

where the set $\partial j \backslash i$ contains all the nearest neighbors of vertex $j$ except for $i$ and $\boldsymbol{c}_{\partial j \backslash i} \equiv\left\{c_{k}: k \in \partial j \backslash i\right\} ; z_{j \rightarrow i}$ is the normalization constant ensuring that $\sum_{c_{i}, c_{j}} q_{j \rightarrow i}^{c_{j}, c_{i}}=1$. This set of equations is collectively referred to as the belief-propagation (BP) equations [26].

Under the Bethe-Peierls approximation the expression for the free energy, $F \equiv-(1 / \beta) \ln Z(\beta)$, of the system is 25,26

$$
F=\sum_{i=1}^{N} f_{i+\partial i}-\sum_{(i, j) \in G} f_{i j},
$$

where $f_{i+\partial i}$ is the contribution of vertex $i$ and all its attached edges, and $f_{i j}$ is the contribution of a single edge $(i, j)$. Because each edge $(i, j)$ contributes to both $f_{i+\partial i}$ and $f_{j+\partial j}$ its effect is subtracted once in Eq. (4). The explicit expressions for $f_{i+\partial i}$ and $f_{i j}$ are:

$$
\begin{gathered}
f_{i+\partial i}=-\frac{1}{\beta} \ln \left[e^{-\beta} \sum_{c_{\partial i}} \Theta\left(\sum_{j \in \partial i} c_{j}-\frac{d_{i}}{2}\right) \prod_{j \in \partial i} q_{j \rightarrow i}^{c_{j}, 1}\right. \\
\left.+\prod_{j \in \partial i}\left(q_{j \rightarrow i}^{0,0}+q_{j \rightarrow i}^{1,0}\right)\right] \\
f_{i j}=-\frac{1}{\beta} \ln \left[\sum_{c_{i}, c_{j}} q_{i \rightarrow j}^{c_{i}, c_{j}} q_{j \rightarrow i}^{c_{j}, c_{i}}\right] .
\end{gathered}
$$

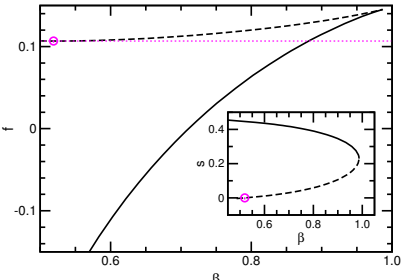

(a)

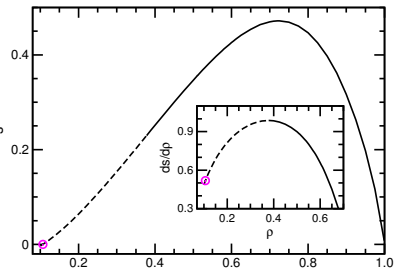

(b)
FIG. 3: The BP equation (3) has two branches of fixedpoint solutions for RR graphs of degree $K=5$. The lowerfree-energy and higher-free-energy branches are drawn as solid and dashed lines, respectively. (a) Free energy density $f$ and entropy density $s$ versus inverse temperature $\beta$. (b) Entropy density $s$ and its first derivative $\mathrm{d} s / \mathrm{d} \rho$ versus energy density $\rho$. The dotted horizontal line and the circles mark the ground-state free energy density and the corresponding $\beta$ and $\rho$ values.

Equations (2)-(6) constitute the replica-symmetric (RS) cavity theory [26] for the SDA problem. For the RR graph ensembles they can be further simplified after considering the vertex uniformity 28. We can iterate the $\mathrm{BP}$ equation either at fixed inverse temperature $\beta$, or at fixed energy density $\rho \equiv \sum_{i=1}^{N} q_{i} / N$ while adjusting $\beta$ [28. The free energy density $f \equiv F / N$ and the entropy density $s \equiv(\rho-f) \beta$ are then computed at a fixed point of BP.

Entropy Inflection.- The results of $f, s$ and $\rho$ for graphs with $K=5$ are shown in Fig. 3, which are representative of all observed RR graphs with $3 \leq K \leq 22$. There is no fixed-point solution in the range of $\beta \geq 0.9866$ ( $K=5$, see another explicit example for $K=3$ in [28]); on the other hand there are two branches of BP fixed points when $\beta$ is smaller, a lower-free-energy (LFE) branch where $f$ increases while both $s$ and $\rho$ decrease with $\beta$, and a higherfree-energy (HFE) branch with opposite behaviors. Both branches are locally stable for fixed $\rho$ (microcanonical ensemble) but unstable with respect to message perturbations at fixed $\beta$ (canonical ensemble) 28]; but because the HFE branch has a higher free energy it cannot be the dominant equilibrium state at a given ambient temperature (the canonical ensemble), even though its energy density $\rho$ is lower. This is a consequence of the much higher entropy of the LFE branch, arguably due to the large number of possible subset selections in larger alliances. The entropy density function $s(\rho)$ is monotonically increasing from zero to the maximum; it is initially convex until an inflection point is reached at $\rho_{x}=0.3775$ with a maximum slope $\beta_{x}=0.9866$. The entropy density approaches zero at $\rho_{o}=0.1067$, indicating that a minimum alliance contains only $0.1067 N$ vertices [35]. The free energy density of the LFE branch exceeds that of the ground state at $\beta_{c}=0.8815$, implying a discontinuous equilibrium phase transition between the high-energy solutions $(\rho \approx 0.511)$ and the ground state $\left(\rho=\rho_{o}\right)$ at this 
critical value $\beta_{c}$ (the corresponding value is $\beta_{c}=0.7491$ for $K=3)$. The predicted discontinuous transition is exactly followed by $\mathrm{SA}$ on the $K=3$ graph instance $\left(\beta_{S A}=\beta_{c}\right.$, Fig. $\left.2 \mathrm{a}\right)$ but it is much delayed by SA on the $K=5$ graph instance $\left(\beta_{S A}>\beta_{c}\right.$, Fig. 2p). Our numerical analysis 28 reveals that the energetic and entropic barriers at the phase transition are finite and low for $K=3,4$ but they are very high for $K=5$. The different SA behaviors of Fig. 2 are consistent with the fact that the minimum SDA problem is easy for $K=3,4$ but NP-hard for $K \geq 5$.

Qualitatively the same theoretical results are obtained for other RR graphs of degree $K \leq 22$ 28. The existence of an inflection point indicates a discrepancy between analyses of the canonical and the microcanonical statistical ensembles 3 [5. Since the slope of $s(\rho)$ defines the intrinsic (microcanonical) inverse temperature, as the temperature $T$ decreases below $1 / \beta_{x}$ the system is no longer capable of finding a matching stable equilibrium and will stay out-of-equilibrium if it has not fortuitously reached a ground state. Notice that entropyinflection is qualitatively different from the temperatureinflection phenomenon of [36] (see also [37]) as the latter does not result in a non-concave entropy curve. Nonconcave microcanonical entropy was also discussed earlier in the contexts of ferromagnetic metastable states [21, 22. and constraint satisfiability problems 38. Interestingly, we find that the entropy density $s(\rho)$ is concave for the entire physical region of $s \geq 0$ (i.e., $\rho \geq \rho_{o}$ ) in high- $K$ graphs $(K \geq 23$, see 28]). In these cases SA indeed successfully finds near-minimum SDA solutions [28].

Message-passing algorithm.- Because of entropy inflection, all configurations of low energy densities $\rho \in\left(\rho_{o}, \rho_{x}\right)$ are invisible in the Boltzmann-Gibbs equilibrium framework where temperature is gradually decreased. It appears that this discontinuity in the equilibrium energy spectrum causes extensive energetic and entropic barriers to the SA dynamics and prohibits the equilibrium transition from the high-energy configurations to the ground states (except the special $K=3,4$ cases for which the barriers are finite [28]). The optimization goal therefore is difficult to accomplish by quasi-equilibrium temperature annealing. One must adopt out-of-equilibrium search strategies. Inspired by the success of mean field theory in exploring the low-energy configuration space we propose a heuristic algorithm termed Clamp-Alliance (CA) for the SDA problem. This algorithm builds on the experiences of earlier message-passing methods $39-$ 43 to perform BP-guided decimation with the objective size of the alliance set $A$ clamped at a low value $n_{o b j}$. At each CA iteration: (1) the cavity probabilities $q_{i \rightarrow j}^{c_{i}, c_{j}}$ are updated several times, with a fine-tuned $\beta$ to ensure fixed mean energy $n_{o b j}$; and (2) the occupation probability for every free vertex is evaluated by Eq. (2), and vertices $i$ with the lowest $q_{i}$ values are deemed unsuitable for alliance membership and are fixed to be non-members
TABLE I: The theoretical minimum energy density $\rho_{o}$ for RR graphs, compared with the mean energy density of alliances obtained by the Clamp-Alliance $\left(\rho_{\mathrm{CA}}\right)$ and simulated annealing $\left(\rho_{\mathrm{SA}}\right)$ algorithms on $50 \mathrm{RR}$ graph instances of size $N=10^{4}$ and degree $K$.

\begin{tabular}{c|l|l|l|l|l|l}
\hline \hline $\mathrm{K}$ & 5 & 6 & 7 & 8 & 9 & 10 \\
\hline$\rho_{o}$ & 0.1067 & 0.0466 & 0.2166 & 0.1430 & 0.2761 & 0.2108 \\
$\rho_{\mathrm{CA}}$ & $0.126(3)$ & $0.062(3)$ & $0.237(2)$ & $0.159(2)$ & $0.295(1)$ & $0.227(2)$ \\
$\rho_{\mathrm{SA}}$ & $0.180(2)$ & $0.101(2)$ & $0.283(2)$ & $0.202(1)$ & $0.334(2)$ & $0.265(1)$ \\
\hline \hline
\end{tabular}

$\left(c_{i}=0\right)$. After the CA iteration stops an initial alliance set will be obtained. This set is then further refined until no other vertices can be removed. More details on the $\mathrm{CA}$ algorithm are provided in [28].

The performance of CA on some RR graphs is demonstrated in Table I. By setting the objective (clamped) alliance size to $n_{o b j} \approx \rho_{o} N$, we see that the solutions obtained by $\mathrm{CA}$ indeed have relative sizes $\rho$ close to the theoretically predicted minimum value $\rho_{o}$. Let us point out that the CA algorithm can also be used to construct a near-minimum alliance set that is associated with a given seed vertex. This latter problem might be particularly relevant for practical applications.

Conclusion and discussion.- We studied a system with bifurcating branches of low and high free-energy configurations within the same temperature range, and revealed a discontinuous phase transition between the highenergy configurations and the non-crystalline ground states. Due to the presence of an inflection point in the entropy-energy profile of the system, the ground states are not associated with a low equilibrium temperature, and simulated annealing generally fails to follow the discontinuous phase transition to reach the ground state. Such a phenomenon is generic to the class of systems with an inflection point, for instance, the presence of a high and a low free-energy branch in the same temperature range is observed in the color-diversity problem studied in 41, but its significance may have been overlooked. Our analyses on entropy inflection are thus crucial as people typically assume a monotonic and concave relation between energy and temperature, but do not verify the concavity property. We introduced an energy-clamping strategy to explore lowest-energy states located in the higher-free-energy branch, which overcomes the limitations of SA. This method can be extended to solve similar problems with a bifurcating configuration space.

The conventional liquid-crystal phase transition is associated with a change in symmetry, but the same does not hold for the present discontinuous phase transition between the high-energy configurations and the ground states, which originates from an inflection point of the entropy-energy profiles. It is interesting to search for such a distinct phase transition in finite-dimensional spin systems.

YZX and CHY contributed equally to this work. Cor- 
respondence should be addressed to HJZ and DS. The following funding supports are acknowledged: Leverhulme Trust Grant RPG-2013-48 (DS); Research Grants Council of Hong Kong Grants 18304316 and 18301217) (CHY); National Natural Science Foundation of China Grants 11421063 and 11747601 (HJZ) and the Chinese Academy of Sciences Grant QYZDJ-SSW-SYS018) (HJZ). Numerical simulations were carried out at the HPC cluster of ITP-CAS and also at the Tianhe-2 platform of the National Supercomputer Center in Guangzhou. We thank Satoshi Takabe for valuable discussions.

[1] N. Metropolis, A. W. Rosenbluth, M. N. Rosenbluth, A. H. Teller, and E. Teller, Equation of state calculations by fast computing machines, J. Chem. Phys. 21, 1087-1092 (1953).

[2] S. Kirkpatrick, C. D. Gelatt, and M. P. Vecchi, Optimization by simulated annealing, Science 220, 671-680 (1983).

[3] H. Touchette, Equivalence and nonequivalence of ensembles: Thermodynamic, macrostate, and measure levels, J. Stat. Phys. 159, 987-1016 (2015).

[4] A. Campa, T. Dauxois, and S. Ruffo, Statistical mechanics and dynamics of solvable models with long-range interactions, Phys. Rep. 480, 57-159 (2009).

[5] D. Mukamel, Statistical mechanics of systems with long range interactions, AIP Conf. Proc. 970, 22 (2008).

[6] B. A. Berg and T. Neuhaus, Multicanonical algorithms for first order phase transitions, Phys. Lett. B 267, 249253 (1991).

[7] C. J. Geyer, Markov chain monte carlo maximum likelihood, In Computing Science and Statistics: Proceedings of the 23rd Symposium on the Interface, pages 156-163, Fairfax Station, Virginia, USA, 1991. Interface Foundation of North America.

[8] A. P. Lyubartsev, A. A. Martsinovski, S. V. Shevkunov, and P. N. Vorontsov-Velyaminov, New approach to monte carlo calculation of the free energy: Method of expanded ensembles, J. Chem. Phys. 96, 1776-1783 (1992).

[9] E. Marinari and G. Parisi, Simulated tempering: a new monte carlo scheme, Europhys. Lett. 19, 451-458 (1992).

[10] K. Hukushima and K. Nemoto, Exchange monte carlo method and application to spin glass simulations, $J$. Phys. Soc. Jpn 65, 1604-1608 (1996).

[11] P. Kristiansen, S. M. Hedetniemi, and S. T. Hedetniemi, Alliances in graphs, J. Combin. Math. Combin. Comput. 48, 157-177 (2004).

[12] M. Jerrum, Large cliques elude the metropolis process, Rand. Struct. Algor. 3, 347-359 (1992).

[13] H. Balakrishnan and N. Deo, Discovering communities in complex networks, In Proceedings of the 44th annual Southeast Regional Conference, pages 280-285. ACM, 2006.

[14] A. Montanari, Finding one community in a sparse graph, J. Stat. Phys. 161, 273-299 (2015).

[15] A. Cami, H. Balakrishnan, N. Deo, and R. D. Dutton, On the complexity of finding optimal global alliances, $J$. Combin. Math. Combin. Comput. 58, 23 (2006).

[16] L. H. Jamieson, S. T. Hedetniemi, and A. A. McRae, The algorithmic complexity of alliances in graphs, J. Combin. Math. Combin. Comput. 68, 137-150 (2009).

[17] I. G. Yero and J. A. Rodríguez-Velázquez, Defensive alliances in graphs: a survey, arXiv:1308.2096 (2013).

[18] C. H. Yeung and D. Saad, Self-sustained clusters and ergodicity breaking in spin models, Phys. Rev. E 88, 032132 (2013).

[19] J. Rocchi, D. Saad, and C. H. Yeung, Self-sustained clusters as drivers of computational hardness in $p$-spin models, Phys. Rev. B 96, 024415 (2017).

[20] J. Rocchi, D. Saad, and C. H. Yeung, Slow spin dynamics and self-sustained clusters in sparsely connected systems, Phys. Rev. E 97, 062154 (2018).

[21] A. Lefèvre and D. S. Dean, Metastable states of a ferromagnet on random thin graphs, Eur. Phys. J. B 21, 121-128 (2001).

[22] A. Pagnani, G. Parisi, and M. Ratiéville, Metastable configurations on the bethe lattice, Phys. Rev. E 67, 026116 (2003).

[23] D. J. Watts, A simple model of global cascades on random networks, Proc. Natl. Acad. Sci. USA 99, 5766-5771 (2002).

[24] M. Mézard, G. Parisi, and M. A. Virasoro, Sk model: the replica solution without replicas, Europhys. Lett. 1, 77-82 (1986).

[25] M. Mézard and G. Parisi, The bethe lattice spin glass revisited, Eur. Phys. J. B 20, 217-233 (2001).

[26] M. Mézard and A. Montanari, Information, Physics, and Computation, Oxford University Press, Oxford, UK, 2009.

[27] G. Araujo-Pardo and L. Barrière, Defensive alliances in regular graphs and circulant graphs, http://hdl.handle.net/2117/2284, 2008.

[28] Supplementary Information notes, which include Ref. 44.

[29] M. E. J. Newman and G. T. Barkema, Monte Carlo Methods in Statistical Physics, Oxford University Press, New York, 1999.

[30] A. Montanari and F. Ricci-Tersenghi, Cooling-schedule dependence of the dynamics of mean-field glasses, Phys. Rev. B 70, 134406 (2004).

[31] F. Krzakala and J. Kurchan, Landscape analysis of constraint satisfaction problems, Phys. Rev. E 76, 021122 (2007).

[32] O. Rivoire, G. Biroli, O. C. Martin, and M. Mézard, Glass models on bethe lattices, Eur. Phys. J. B 37, 55-78 (2004).

[33] M. Mézard and A. Montanari, Reconstruction on trees and spin glass transition, J. Stat. Phys. 124, 1317-1350 (2006).

[34] F. Krzakala, A. Montanari, F. Ricci-Tersenghi, G. Semerjian, and L. Zdeborová, Gibbs states and the set of solutions of random constraint satisfaction problems, Proc. Natl. Acad. Sci. USA 104, 10318-10323 (2007).

[35] Y. Kabashima and D. Saad, Statistical mechanics of error-correcting codes, Europhys. Lett. 45, 97-103 (1999).

[36] S. Schnabel, D. T. Seaton, D. P. Landau, and M. Bachmann, Microcanonical entropy inlection points: Key to systematic understanding of transitions in finite systems, Phys. Rev. E 84, 011127 (2011).

[37] K. Qi and M. Bachmann, Classification of phase transitions by microcanonical inflection-point analysis, Phys. Rev. Lett. 120, 180601 (2018). 
[38] H.-J. Zhou and C. Wang, Ground-state configuration space heterogeneity of random finite-connectivity spin glasses and random constraint satisfaction problems, $J$. Stat. Mech.: Theor. Exp., P10010 (2010).

[39] M. Mézard, G. Parisi, and R. Zecchina, Analytic and algorithmic solution of random satisfiability problems, Science 297, 812-815 (2002).

[40] A. Montanari, F. Ricci-Tersenghi, and G. Semerjian, Solving constraint satisfaction problems through belief propagation-guided decimation, In Proceedings of 45th Annual Allerton Conference on Communication, Control, and Computing, pages 352-359, New York, 2007. Curran Associates, Inc.
[41] K. Y. M. Wong and D. Saad, Minimizing unsatisfaction in colourful neighbourhoods, J. Phys. A: Math. Theor. 41, 324023 (2008).

[42] P. Sulc and L. Zdeborová, Belief propagation for graph partitioning, J. Phys. A: Math. Theor. 43, 285003 (2010).

[43] Y.-Z. Xu and H.-J. Zhou, Optimal segmentation of directed graph and the minimum number of feedback arcs, J. Stat. Phys. 169, 187-202 (2017).

[44] E. Marinari and R. Monasson. Circuits in random graphs: from local trees to global loops. J. Stat. Mech.: Theory Exper., P09004 (2004). 